\title{
Clinical features and temporal changes of RT-PCR and chest CT in COVID-19 pediatric patients
}

\author{
Wei Xia ${ }^{1}, Y_{u}$ Guo $^{1}$, Zhiyao Tian ${ }^{2}$, Yan $\mathrm{Luo}^{3}$, Daoyu Hu${ }^{4}$, Jianbo Shao ${ }^{1}$, Zhen $\mathrm{Li}^{3}$, and Ihab \\ R Kamel ${ }^{5}$ \\ ${ }^{1}$ Wuhan Childrens Hospital \\ ${ }^{2}$ Wuhan Children's Hospital (Wuhan Maternal and Child Healthcare Hospital), Tongji \\ Medical College, Huazhong University of Science and Technology \\ ${ }^{3}$ Tongji Hospital, Tongji Medical college, Huazhong University of Science and Technology \\ ${ }^{4}$ Tongji Hospital of Tongji Medical College of Huazhong University of Science and \\ Technology \\ ${ }^{5}$ Russell H Morgan Department of Radiology \& Radiological Sciences, Johns Hopkins \\ Hospital, Johns Hopkins Med Inst
}

June 10, 2020

\begin{abstract}
Purpose: To investigate the clinical features and temporal changes of RT-PCR and CT in COVID-19 pediatric patients. Methods: Clinical, RT-PCR and CT features of 114 COVID-19 pediatric inpatients were retrospectively reviewed from January 21st to March 14th 2020. All patients had chest CT on admission and were identified as positive pharyngeal swab nucleic acid test. Clinical features were analyzed, as well as the features and temporal changes of RT-PCR and CT. Results: Fever (62, $54 \%)$ and cough $(61,54 \%)$ were the most common symptoms. There were $34(30 \%)$ cases of concurrent infections. The most common imaging features on CT were ground-glass opacities (46, 40\%) and consolidation (46, 40\%). The bilateral lower lobes were the most common pattern of involvement, with 63 cases (55\%) involving 1-2 lobes, and in 32 (28\%) cases CT was normal. Through the whole duration of COVID-19 in children, the diagnostic positive rate of RT-PCR has been far higher than that of CT (All $\mathrm{P}_{i} \cdot 05$ ). For RT-PCR follow-up, reliable negative results were obtained only 7 days after the onset of symptoms. Though Lung involvement on chest CT progressed rapidly in several cases, lung involvement in children with COVID-19 is mild, with a median value of 2 on $\mathrm{CT}$ score. Conclusion: RT-PCR is more reliable than $\mathrm{CT}$ in initial diagnosis of pediatric patients with COVID-19. On follow up, reliable RT-PCR results are available 7 days after initial symptoms. Only if necessary, CT could be used for follow-up.
\end{abstract}

\section{Introduction}

Since December 2019, a newly discovered infectious disease named COVID-19 caused by a novel coronavirus (SARS-CoV-2) has wildly spread worldwide. More than six million COVID-19 patients have been confirmed, and thousands of children are also involved in this pandemic all over the world ${ }^{1-3}$.

To date, viral nucleic acid testing and chest CT have been considered as the main diagnostic methods for patients with suspected COVID-19 pneumonia. However, in adults, the positive rate of pharyngeal swabs reverse transcription polymerase chain reaction (RT-PCR) is only $59 \%$ to $61 \cdot 3 \%^{4,5}$. For adults, despite the possibility of false positives, the positive rate of chest CT is significantly higher than that of RT-PCR and is considered a more accurate early diagnostic tool ${ }^{6-9}$.

For pediatric patients with suspected COVID-19, studies on chest CT values are limited ${ }^{10,11}$. Some studies 
have suggested that normal CT or lack of typical features were not uncommon in pediatric patients, especially during the early stage of the diseases ${ }^{12-15}$. The most common CT feature of pediatric patients was bilateral ground-glass opacities with a ratio of only $32.7 \%^{12}$. However, the use of CT should be carefully assessed since pediatric patients are sensitive to radiation, and reducing CT scans for children is a top priority.

Compared with adults, the risk of severe or fatal COVID-19 disease is rare while the majority of them were mild cases requiring only conventional therapy for viral pneumonia ${ }^{12-14}$. In addition, viral infections, such as influenza virus, coxsackie virus, rubella virus and $\mathrm{EB}$ virus, in pediatric patients are very common, so pediatricians often do not initially suspect a diagnosis of COVID-19. However, during this pandemic, this will lead to missed diagnosis of COVID-19 and increase the likelihood of further transmission of the disease from undiagnosed children to healthy individuals.

In this pandemic emergency, it is important to optimize limited medical resources, reduce the radiation dose for children and obtain a rapid and accurate diagnosis. Thus, we performed a longitudinal study to analyze the clinical characteristics, CT manifestations, and RT-PCR changes to explore early diagnosis strategies for pediatric patients.

\section{Materials and methods}

\subsection{Study design and participants}

The study was performed in accordance with the Declaration of Helsinki principles and Good Clinical Practice. The study protocol was approved by the Institutional Review Board of Wuhan Children's Hospital and Tongji Hospital, Tongji Medical College, Huazhong University of Science and Technology (Wuhan, China). Written informed consent was waived because of the emergence of this infectious disease.

Medical record review was conducted using the institution's database from January $21^{\text {st }}$ to March $14^{\text {th }} 2020$ in Wuhan Children's hospital and Tongji Hospital. In a prior study included only 20 patients in Wuhan children's hospital, it only described the general clinical and CT features without follow-up, and didn't include the evaluation of RT-PCR ${ }^{13}$.

Inclusion criteria were: (a) age ¡16 Years old, inpatients; (b) positive RT-PCR results of COVID-19; (c) CT in the picture archiving and communication system (PACS) performed. (d) discharged patients only.

Exclusion criteria were: (a) CT scans were performed greater than 1 months; (b) patients with suboptimal image quality for analysis due to breathing motion artifact; (c) unknown date of RT-PCR.

Pharyngeal swab samples of all the pediatric patients in our study were collected, and the SARS-CoV2 RNA was detected by RT-PCR. RT-PCR kits from Wuhan HUADA biotechnology Co., Ltd, Shanghai Huirui Biotechnology Co., Ltd or Shanghai BioGerm Medical Biotechnology Co., Ltd. These were approved by China Food and Drug Administration for the detection of SARS-CoV-2 nucleic acid.

The discharge criteria were as follows: (1) normal temperature for at least 3 days; (2) significantly improved respiratory symptoms; (3) two consecutive SARS-CoV-2 throat swabs with negative RT-PCR results were performed at least 24 hours apart.

\subsection{Chest CT protocols}

Non-enhanced chest CT were performed in either of the four CT units (SOMATOM Definition AS128, Siemens; uCT 780, United Imaging; Optima 660, GE; SOMATOM Definition AS+, Siemens) with the slice thickness of $10 \mathrm{~mm}$. The scanning range covered from lung apex to diaphragm on axial plane taken under free breathing with the patients in supine position. If necessary, $0^{*} 50 \mathrm{ml} / \mathrm{kg}$ body mass of $10 \%$ chloral hydrate was taken orally before the examination. Thin-section CT images were reconstructed with $0^{*} 625 \mathrm{~mm}$ collimation with a standard algorithm and then sent to the Picture Archiving and Communication System (PACS) for analyzing.

\subsection{Data collection and analysis}


We reviewed the clinical charts of all the pediatric patients for demographic information, symptoms, date of symptom onset, admission date, discharge date, dates and results of nucleic acid tests for COVID-19, and other identified concurrent infectious pathogens.

Two radiologists (WX and ZL with 12 and 18 years of experience, respectively) independently reviewed the chest CT images on PACS, only decision reached in consensus was reported. The initial CT images were stratified into one of two groups: normal or abnormal group. The CT images in the abnormal group were further assessed for imaging features including: (a) unilateral or bilateral distribution, (b) lobes involved, and (c) lesion characteristics. Lesion characteristics were subcategorized into: (a) ground-glass opacity, (b) consolidation, (c) nodule, and (d) thickened interlobular septa.

The involvement of lung was quantified according to a previously published paper, which had applied it in adults with COVID-19 ${ }^{16}$. We divided each lung into upper zone (above the carina), middle zone, and lower zone (below the inferior pulmonary vein). And each zone was scored for percentage of lung involved on a scale of 0-4 ( 0 for $0 \%$ involvement; 1 for less than $25 \%$ involvement; 2 for $25 \%$ to less than $50 \%$ involvement; 3 for $50 \%$ to less than $75 \%$; 4 for more than $75 \%$ involvement). Overall CT score of lung involvement was the summation of scores from all six lung zones.

\subsection{Statistics}

Statistical analysis was performed using SPSS 19 (IBM Corporation, NY, USA). The day of onset of symptom was set as day 0 . The cumulative percentages of cases diagnosed by RT-PCR and CT as a function of time were plotted separately. Following initial positive RT-PCR, the cumulative percentages on first negative results and final negative results of follow-up RT-PCR were plotted as well. The CT scores of lung involvement were plotted over time. The cumulative cases diagnosed by RT-PCR and CT at different time points were compared by Chi-square test, two-tailed, and $P i^{*} 05$ was considered statistically significant difference, as well as for the comparison of chest CT features between cases with and without coinfection.

\section{Results}

Between Jan. $21^{\text {st }}$ and Mar. $14^{\text {th }}, 2020$, the total number of RT-PCR confirmed COVID-19 pediatric discharged patients who has undergone CT was one hundred and twenty-three. Of those, nine cases with unknown date of RT-PCR were excluded. The final number of patients included in this cohort was 114. All included patients were residents of Wuhan. The flow chart was shown in E-figure 1.

\subsection{Clinical characteristics}

The demographics and clinical characteristics of all the patients were summarized in Table 1. Male (69, 61\%) and school-age children (older than 6 years, 56, 49\%) were more susceptible to COVID-19. In most cases, the duration from symptom onset to discharge was less than 21 days $(87,76 \%$ ), with an average hospital stay of 13 days. Fever $(62,54 \%)$ and cough $(61,54 \%)$ were the most common symptoms. Concurrent infection were found in $34(30 \%)$ cases, with mycoplasma $(29,25 \%)$ being the most common concurrent infectious pathogen.

\subsection{Chest CT features}

The characteristics of chest CT were reported in Table 2. During the hospitalization period, ninety-seven (85\%) pediatric patients were performed no more than two chest CTs, while $17(15 \%)$ cases were performed 3 or more CT scans. During all the patients, normal chest CTs were found in $32(28 \%)$ cases, and no more than 2 lobes involved in $63(55 \%)$ cases. Unilateral lung involvement was found in $46(40 \%)$ cases, and bilateral lung involvement was found in $36(32 \%)$ cases. Bilateral inferior lobes were most susceptible to COVID-19, with 43 cases (38\%) affected in both the left and right lower lobe, respectively. Ground-glass opacity $(46,40 \%)$ and consolidation $(46,40 \%)$ were the most common lesion patterns (E-figures 2 and 3 for details), while nodules were found in 7 (6\%) cases (E-figure 4 for details). A comparison of chest CT features between cases with and without coinfection was shown in E-table 1.

\subsection{RT-PCR characteristics}


Detailed information of RT-PCR was displayed in Table 3. All the cases have 3 to 12 times RT-PCR during their hospital stay, and $89(77 \%)$ cases have 5 or less RT-PCR assays. The duration from first positive result to final negative result was within 21 days in 105 (92\%) cases. The most common temporal tendency pattern of RT-PCR was positive to negative $(91,80 \%)$.

\subsection{Temporal changes of initial chest CT and RT-PCR}

The cumulative percentage of cases identified by RT-PCR and initial chest CT is shown in Figure 1. In the first week after the onset of symptoms, the cumulative percentage of RT-PCR and CT both increased rapidly. On the 4th day after the onset of symptoms, 72 (63\%) patients had a positive RT-PCR, but only 36 (42\%) patients had a lung invasion in the chest $\mathrm{CT}$ at the same time $\left(\chi^{2}=22 \cdot 8, P i^{*} 05\right)$. On the seventh day after the onset of symptoms, RT-PCR confirmed 89 cases (78\%) of COVID-19 pneumonia, while chest CT confirmed only 53 cases $(46 \%)\left(\chi^{2}=24 \cdot 2, P i^{*} 05\right)$. By the 14 th day of symptom onset, 112 cases $(98 \%)$ were confirmed by RT-PCR, but only 76 cases $(67 \%)$ were positive for chest CT $\left(\chi^{2}=39 \cdot 3, P i^{*} 05\right)$. Eighteen days after the onset of symptoms, the last patient was confirmed by RT-PCR, comparing to 77 (68\%) identified cases by CT $\left(\chi^{2}=44 \cdot 2, P i^{*} 05\right)$. As the chest CT of 32 children was normal, the cumulative percentage of cases identified by CT was only $72 \%$ (82), comparing to $114(100 \%)$ cases identified by RT-PCR $\left(\chi^{2}=37 \cdot 2\right.$, $\left.P i^{*} 05\right)$. Through the whole duration of COVID-19 in children, the diagnostic positive rate of RT-PCR has been far higher than that of $\mathrm{CT}$ (All $\left.\mathrm{P}_{i}^{*} 05\right)$, as shown in E-table 2.

At the same time, it was found that after the first week of symptoms, the rise of cumulative percentage of cases identified by RT-PCR and chest CT became less, and reached a stable level after 11 days. Compared with the initial chest CT, the cumulative percentage of cases identified by RT-PCR is more significant.

\subsection{Temporal changes of RT-PCR and chest CT}

For treatment responds, a follow-up review of the treatment responds of RT-PCR for children indicated that (Figure 2), there were no truly negative RT-PCR results until 7 days after the onset of symptoms. There were $52(46 \%)$ truly negative RT-PCR results cases on the 14th day, $92(81 \%)$ cases on the 21st day, and $106(93 \%)$ cases on the 28 th day, and the latest time for RT-PCR to turn negative was 37 days after the onset of symptoms.

A total of 215 chest CTs were obtained from 114 children with COVID-19. In Figure 3, the CT scores on lung involvement were plotted in function of time. CT score reached 14 in 1 case during the 0-5 days from the onset of symptoms, but the median values of CT scores were low before the 11th day from the onset of symptoms, with the median value of 1 . The median value of CT score on lung involvement reached a peak at 12-17 days, with the median value of 2 . After the 24 th day from onset of symptoms, the median value of CT score fell back to 1 .

\section{Discussion}

In the current study, the length of hospital stay in most pediatric patients with COVID-19 (average 13 days) was not significantly different from pneumonia caused by other pathogens (average $11^{*} 2$ days) ${ }^{17}$. Interestingly, the number of pediatric patients younger than 6 months $(11,10 \%)$ was significantly less than that in other age groups, which may be related to residual protection from maternal immune factors. The number of patients in other two age groups (6 Months to 6 Ys, 47, 41\%; >6Ys, 56, 49\%) was not significantly different from each other. Similar to results reported in previous studies, according to the lung involvement, the majority of cases were mild cases and only conventional therapy for viral pneumonia was needed ${ }^{12,13,18}$. Severe and fatal cases in pediatric patients were very rare. Thus, early diagnosis to avoid further spread of disease would be much more important than treatment for individuals.

In the current study, chest CT had similar characteristic manifestations including ground-glass opacities and consolidations with bilateral inferior lobes, as was recently published ${ }^{13,19}$. Of all 114 COVID-19 positive children in this study, $32(28 \%)$ had completely normal chest CT, $63(55 \%)$ had no more than 2 lung lobe lesions, and 34 patients had concurrent infections (30\%). Similar to previous studies, CT of the chest is often atypical, especially in the early stage, resulting in difficulty in diagnosing or ruling out COVID-19 ${ }^{20}$. 
In addition, in our study chest CT identified only 53 (46\%) cases by day 7 of the onset of symptoms, and by day 14 , only $76(67 \%)$ cases of chest CT were positive. This is totally different from the results of related research in adults. In adults, as an important complementary tool for low-sensitive and time-consuming RT-PCR, chest CT has first been considered as diagnostic tool for clinical confirmed case of COVID-19 in China, where the epidemic is most severe ${ }^{21,22}$. Considering that the clinical manifestations and CT features of most children are mild, CT has limited diagnostic value for children (especially 0-7 days after onset). Therefore, CT of the chest is of limited value in early stages of the disease, and should be discouraged to reduce the radiation dose to children.

In the current study, throughout the entire course of COVID-19 pneumonia in children, the diagnostic positive rate of $\mathrm{CT}$ has been far lower than that of RT-PCR (All $P i^{*} 05$ ), and $28 \%$ of children have no obvious abnormal signs of CT. However, in adults, previous studies have shown that the positive rate of RT-PCR is only $59 \%$ to $61^{*} 3 \%$, while the positive rate of chest CT is $88 \%^{4,5}$. These findings suggest that most infected pediatric patients have less lung involvement in the early stages of the COVID-19 infection. As some COVID-19 cases confirmed by RT-PCR could have no lesions on chest CT, pathogen identification by RT-PCR have more important role in management of infectious source. RT-PCR maybe is more reliable than CT in pediatric patients' diagnosis, and repeated RT-PCR is the recommended screening for pediatric patients during the first 7 days.

In addition, the main indicator of children's recovery is to determine the true RT-PCR negative examination results. For well-known reasons, we may inevitably get false negative RT-PCR results in treatment responds evaluation. Our research shows that only RT-PCR results obtained 7 days after the onset of symptoms are reliable. This indicates that for children who have disappeared clinical symptoms and may totally recover, the follow-up tests of RT-PCR to evaluate the efficacy must be performed at least after day 7.

To reduce radiation dose of pediatric patients included in this study, most cases (85\%) had 1 or 2 chest CT scans during their hospital stay. In our study, it was found that the median values of CT scores were low before the 11th day from the onset of symptoms, with the median value of 1 . The median value of CT score on lung involvement reached a peak at 12-17 days, with the median value of 2. After the 24th day from onset of symptoms, the median value of CT score fell back to 1 . Comparing to median CT score of 5 on lung involvement in adults reported in previous study ${ }^{16}$, it indicates that children with COVID-19 pneumonia are relatively mild. As reported, lung involvement peaking on 6 to 11 days from symptom onset in adults, delayed peaking in children may be related to different immune reaction to virus ${ }^{16}$. In our study, CT score reached 14 in 1 case during the 0-5 days from the onset of symptoms, which suggested that rapid progression could also be observed in pediatric patients, even if it was rare.

There are several limitations in our study. First, even if the sample was the largest as we known, the overall cases in the two included hospitals was still limited. Second, as a retrospective study, selection bias could not be avoided. Third, during this outbreak period of COVID-19, delay in seeking care (more than 7 days from symptom onset) would influence the diagnosis and prognosis.

In conclusion, chest $\mathrm{CT}$ is not recommended as a primary method for early diagnosis in children with COVID-19, especially to avoid repeated CT scans, while RT-PCR may have a more valuable position. For treatment responds, reliable RT-PCR follow-up results are not available until at least 7 days after the onset of symptoms. Only if necessary, CT can be employed as a tool to assess lung involvement.

\section{Competing Interests}

The authors have declared that no competing interest exists.

\section{References}

1. Huang C, Wang Y, Li X, Ren L, Zhao J, Hu Y, Zhang L, Fan G, Xu J, Gu X, et al. Clinical features of patients infected with 2019 novel coronavirus in Wuhan, China. Lancet 2020;395(10223):497-506.

2. Guan W-j, Ni Z-y, Hu Y, Liang W-h, Ou C-q, He J-x, Liu L, Shan H, Lei C-l, Hui DSC, et al. Clinical 
Characteristics of Coronavirus Disease 2019 in China. N Engl J Med2020.

3. Kandel N, Chungong S, Omaar A, Xing J. Health security capacities in the context of COVID-19 outbreak: an analysis of International Health Regulations annual report data from 182 countries. The Lancet: Elsevier; 2020 .

4. Ai T, Yang Z, Hou H, Zhan C, Chen C, Lv W, Tao Q, Sun Z, Xia L. Correlation of Chest CT and RT-PCR Testing in Coronavirus Disease 2019 (COVID-19) in China: A Report of 1014 Cases. Radiology. Volume 02020. p 200642.

5. Yang Y, Yang M, Shen C, Wang F, Yuan J, Li J, Zhang M, Wang Z, Xing L, Wei J, et al. Evaluating the accuracy of different respiratory specimens in the laboratory diagnosis and monitoring the viral shedding of 2019-nCoV infections. medRxiv2020. p 2020.2002.2011.20021493.

6. Bernheim A, Mei X, Huang M, Yang Y, Fayad ZA, Zhang N, Diao K, Lin B, Zhu X, Li K, et al. Chest CT Findings in Coronavirus Disease-19 (COVID-19): Relationship to Duration of Infection. Radiology. Volume 02020. p 200463.

7. Fang Y, Zhang H, Xie J, Lin M, Ying L, Pang P, Ji W. Sensitivity of Chest CT for COVID-19: Comparison to RT-PCR. Radiology. Volume 02020. p 200432.

8. Pan F, Ye T, Sun P, Gui S, Liang B, Li L, Zheng D, Wang J, Hesketh RL, Yang L, et al. Time Course of Lung Changes On Chest CT During Recovery From 2019 Novel Coronavirus (COVID-19) Pneumonia. Radiology. Volume 02020. p 200370.

9. Xie X, Zhong Z, Zhao W, Zheng C, Wang F, Liu J. Chest CT for Typical 2019-nCoV Pneumonia: Relationship to Negative RT-PCR Testing. Radiology. Volume 02020. p 200343.

10. Wu Z, McGoogan JM. Characteristics of and Important Lessons From the Coronavirus Disease 2019 (COVID-19) Outbreak in China: Summary of a Report of 72314 Cases From the Chinese Center for Disease Control and Prevention. JAMA2020.

11. Guan WJ, Ni ZY, Hu Y, Liang WH, Ou CQ, He JX, Liu L, Shan H, Lei CL, Hui DSC, et al. Clinical Characteristics of Coronavirus Disease 2019 in China. N Engl J Med. February 28 ed2020.

12. Lu X, Zhang L, Du H, Zhang J, Li YY, Qu J, Zhang W, Wang Y, Bao S, Li Y, et al. SARS-CoV-2 Infection in Children. N Engl J Med. published online March 18 ed2020.

13. Xia W, Shao J, Guo Y, Peng X, Li Z, Hu D. Clinical and CT features in pediatric patients with COVID-19 infection: Different points from adults. Pediatr Pulmonol2020.

14. Liu W, Zhang Q, Chen J, Xiang R, Song H, Shu S, Chen L, Liang L, Zhou J, You L, et al. Detection of Covid-19 in Children in Early January 2020 in Wuhan, China. N Engl J Med2020.

15. Yu N, Li W, Kang Q, Xiong Z, Wang S, Lin X, Liu Y, Xiao J, Liu H, Deng D, et al. Clinical features and obstetric and neonatal outcomes of pregnant patients with COVID-19 in Wuhan, China: a retrospective, single-centre, descriptive study. The Lancet Infectious Diseases: Elsevier; 2020.

16. Wang Y, Dong C, Hu Y, Li C, Ren Q, Zhang X, Shi H, Zhou M. Temporal Changes of CT Findings in 90 Patients with COVID-19 Pneumonia: A Longitudinal Study. Radiology. Volume 02020. p 200843.

17. Wen ZH, Wei J, Xue HL, Chen YQ, Melnick D, Gonzalez J, Hackett J, Li XY, Cao ZL. Epidemiology, microbiology, and treatment patterns of pediatric patients hospitalized with pneumonia at two hospitals in China: a patient chart review study. Ther Clin Risk Manag 2018;14:501-510.

18. Zhu N, Zhang D, Wang W, Li X, Yang B, Song J, Zhao X, Huang B, Shi W, Lu R, et al. A Novel Coronavirus from Patients with Pneumonia in China, 2019. N Engl J Med 2020;382(8):727-733.

19. Chung M, Bernheim A, Mei X, Zhang N, Huang M, Zeng X, Cui J, Xu W, Yang Y, Fayad Z, et al. CT Imaging Features of 2019 Novel Coronavirus (2019-nCoV). Radiology. Volume 2952020. p 200230-200230. 
20. Virkki R, Juven T, Rikalainen H, Svedstrom E, Mertsola J, Ruuskanen O. Differentiation of bacterial and viral pneumonia in children. Thorax 2002;57(5):438-441.

21. General Office of the National Health Commission of China. Diagnosis and Treatment Protocol for 2019-nCoV (Fifth Trial Edition). Fifth Trial Edition ed. Volume 20202020.

22. Yang W, Yan F. Patients with RT-PCR Confirmed COVID-19 and Normal Chest CT. Radiology. published online ed. Volume 02020. p 200702.
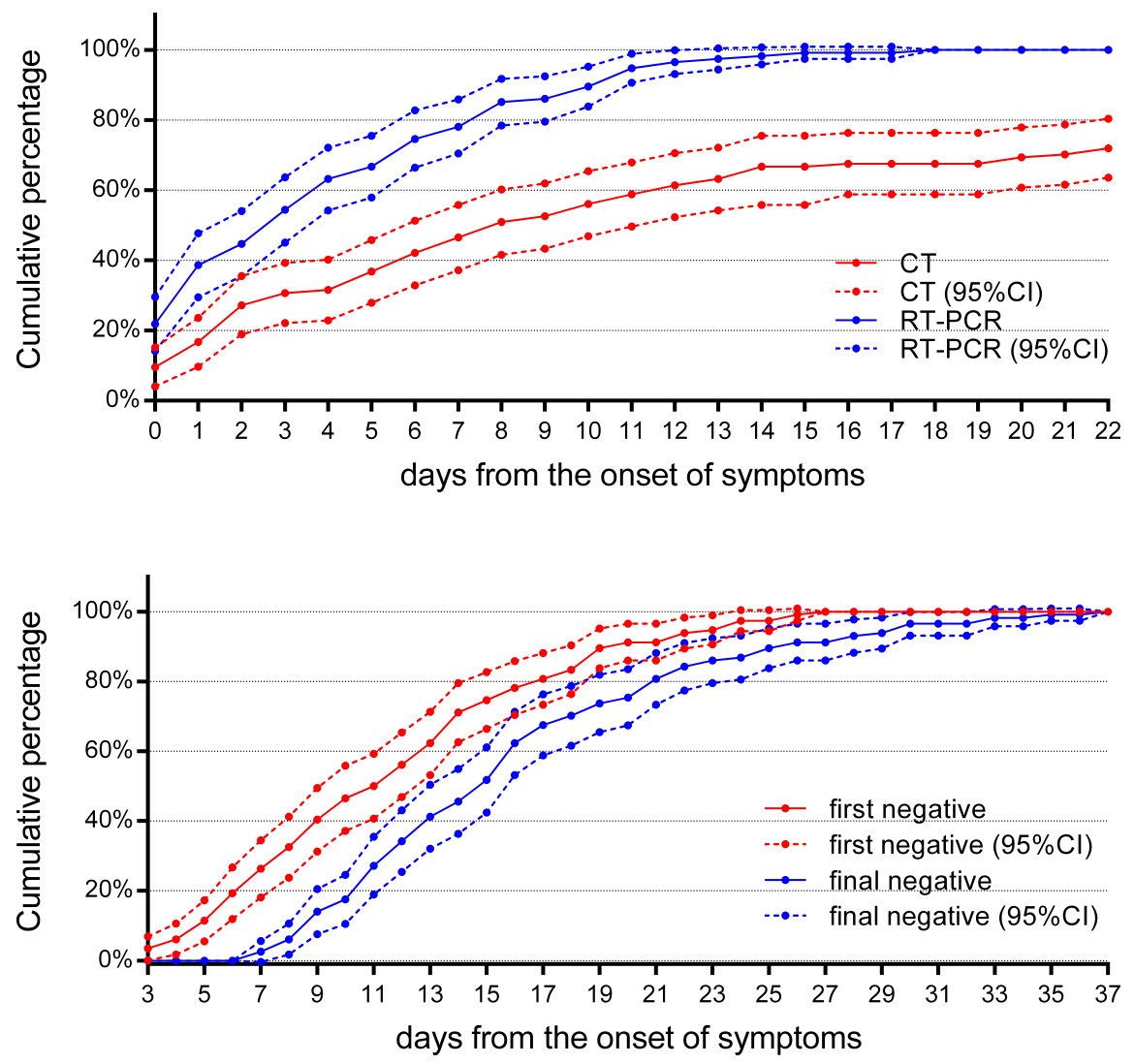


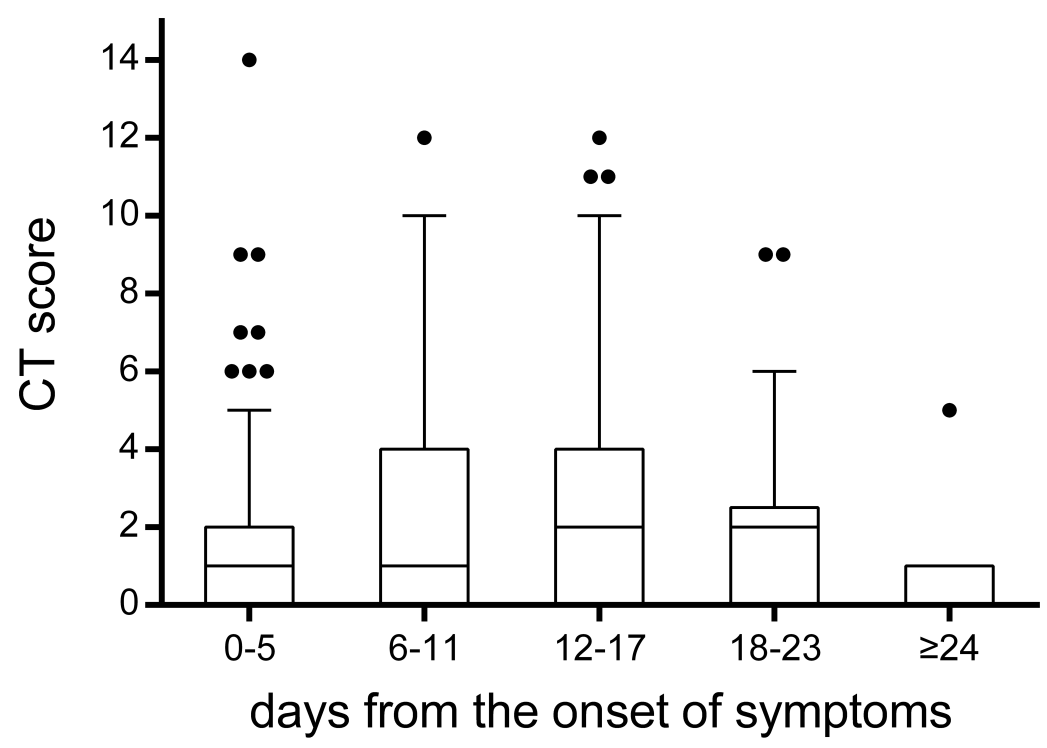

\section{Hosted file}

table609.docx available at https://authorea.com/users/332097/articles/458543-clinicalfeatures-and-temporal-changes-of-rt-pcr-and-chest-ct-in-covid-19-pediatric-patients 